\title{
Control Problems for One-Dimensional Fluids and Reactive Fluids with Moving Interfaces
}

Nicolas Petit

\begin{abstract}
The purpose of this paper is to expose several recent challenging control problems for mono-dimensional fluids or reactive fluids. These problems have in common the existence of a moving interface separating two spatial zones where the dynamics are rather different. All these problems are grounded on topics of engineering interest. The aim of the author is to expose the main control issues, possible solutions and to spur an interest for other future contributors. As will appear, mobile interfaces play key roles in various problems, and truly capture main phenomena at stake in the dynamics of the considered systems.
\end{abstract}

\section{Introduction}

The purpose of this paper is to expose several recent challenging control problems for mono-dimensional fluids or reactive fluids. These problems have in common the existence of a moving interface separating two spatial zones where the dynamics are rather different. All these problems are grounded on topics of engineering interest. The aim of the author is to expose the main control issues, possible solutions and to spur an interest for other future contributors. As will appear, mobile interfaces play key roles in various problems, and truly capture main phenomena at stake in the dynamics of the considered systems.

The paper contains a brief panorama. It is organized as follows. In Section 2, a Diesel oxidation catalyst for the automotive industry is considered. A boundary control problem is formulated for the outlet temperature control

Nicolas Petit

MINES ParisTech, Centre Automatique et Systèmes,

Unité Mathématiques et Systèmes

60, boulevard Saint-Michel, 75272 Paris, France

e-mail: nicolas.petit@mines-paristech.fr

http://cas.ensmp.fr/ petit

J. Lévine \& P. Müllhaupt (Eds.): Adv. in Theory of Control, Signals, LNCIS 407, pp. $323-337$. springerlink.com

(C) Springer-Verlag Berlin Heidelberg 2010 
of this distributed reactive gaseous system. A model mobile interface separates the upstream reactive zone from the downstream transport zone. The location of this frontier is dependent on several variables, including measured disturbances and the control variable. In Section 3, a classic Stefan problem is presented. This system represents the melting of a solid phase into a liquid phase which is heated on its boundary. Heat propagates inside the system and generates a melting which occurs at a distance from the heat source which varies as the solid phase melts or grows. As will appear, both the temperature and the location of the moving interface can be controlled by the boundary actuation. In Section 4, some mixing models for stirring vessels are exposed. The proposed models use a mobile interface separating a distributed plug flow regime from a continuously stirred homogenous zone. The motion of the interface is generated by the variations of the blending speed which is a control variable. Finally, in Section 5, some recent developments on multiphasis slug flow are exposed. They appear in the petroleum industry. Slugs are large bubbles of gas separating pockets of liquid. They appear under certain flow conditions, and must be avoided as they have malicious effects. Models for them, involving a virtual choke which plays the role of a controlled interface, are discussed.

\section{Diesel Oxidation Catalyst}

This introductory example comes from the automotive engine control world. On most modern diesel vehicles, the increasing requirements regarding particulate matter emissions are satisfied using a particulate filter (DPF). This device is now widely spread among new vehicles. The filter, located in the vehicle exhaust line, stores particulate matter until it is burnt during an active regeneration process. This regeneration is achieved by raising the filter temperature (between 450 and 600 degrees) in the presence of oxygen in a diesel oxidation catalyst (DOC).

Historically, oxidation catalysts have been the first aftertreatment systems in the automotive industry. Catalysts used for diesel applications have appeared only recently because of the relative lower values of hydrocarbon reductants $\mathrm{HC}$ and $\mathrm{CO}$ emissions found in compression ignition engines compared against spark ignition engines. Because the $\mathrm{HC}$ and $\mathrm{CO}$ reactions are strongly exothermic, the DOC is also used to control the exhaust line temperature. In particular, it is used to generate the temperature required for the already mentioned DPF active regeneration. To increase the DPF inlet temperature, reductants are oxidized inside the DOC, which, in turn, increases its outlet temperature.

After treatment systems use monolith converters which are designed to maximize the mass transfer to the catalytic surface. To this end, the channels of the monolith are narrow and numerous (a typical order of magnitude is 
400 cpsi). This geometric configuration (see Figure1) also yields highly-efficient heat transfer between gas and solid. Hence, the solid phase (i.e. the monolith) acts as a spatially-distributed storage of energy and species. As can be experimentally observed, the induced propagation phenomenon leads to highlydelayed responses. Models for these devices are based on one-dimensional distributed parameter equations. These one-dimensional effects must be included in the modeling and further, they must be accounted for in the control strategies if performance is desired.

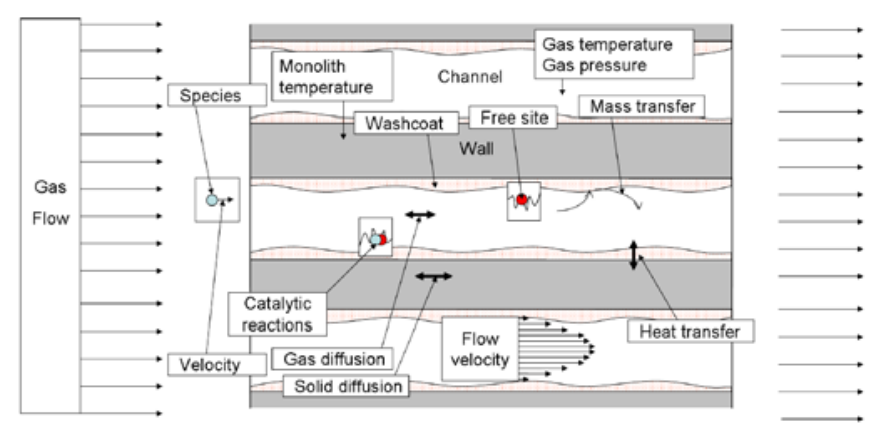

Fig. 1. Phenomena involved in the numerous channels of a Diesel Oxidation Catalyst. Reductant species in the exhaust gas are converted on the distributed catalyst surface.

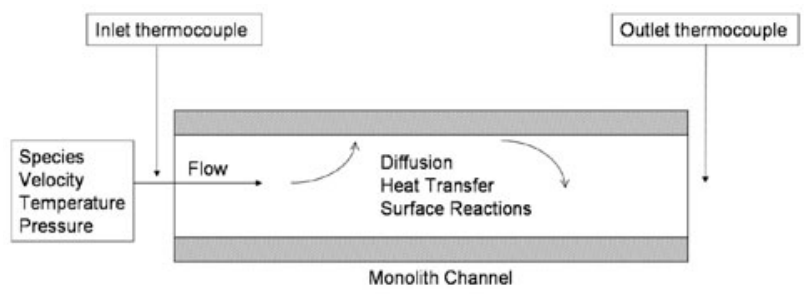

Fig. 2. Scheme of governing phenomena in a Diesel Oxidation Catalyst

Considering thermal effects, a simple model for the DOC consists of the following balance equations

$$
\begin{aligned}
\frac{\partial T}{\partial t}+v \frac{\partial T}{\partial z} & =-k_{1}\left(T-T_{s}\right) \\
\frac{\partial T_{s}}{\partial z} & =k_{2}\left(T-T_{s}\right)
\end{aligned}
$$

which represent the dynamics of the temperature of the gas $(T)$ and the temperature of the monolith $\left(T_{s}\right)$. These equations are pictured in Figure 2 . The control variable is the inlet temperature 


$$
T(0, t)=u(t)
$$

Mathematically, this system of equations has a surprisingly long response time, which is consistent with experimental observations 16 discussed earlier.

In details, the input-output relationship can be easily calculated from the following transfer function (in the Laplace domain)

$$
\hat{T}(z, s)=\hat{u}(s) \exp \left(-\frac{z}{v} s-\frac{k_{1} z}{v}+\frac{m}{s+k_{2}}\right)
$$

which gives, in the time-domain,

$$
\begin{aligned}
T(z, t)= & H\left(t-\frac{z}{v}\right) \exp \left(-\frac{k_{1} z}{v}\right) \\
& \times\left(u\left(t-\frac{z}{v}\right)+\int_{0}^{t-\frac{z}{v}} \exp \left(-k_{2} \tau\right) \sqrt{\frac{m}{\tau}} I_{1}(2 \sqrt{m \tau}) u\left(t-\frac{z}{v}-\tau\right) d \tau\right)
\end{aligned}
$$

where $H$ is the Heaviside function. The above formula, which involves a modified Bessel function, kindly fits experimental data, as can be observed in Figure 3 .

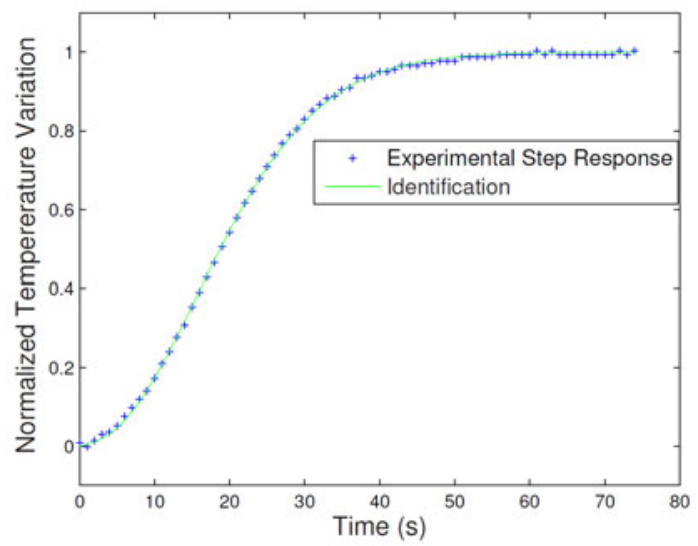

Fig. 3. Experimental data versus the DOC model

For (open-loop) control design, the transfer function (3) can be readily inverted. This gives

$$
\hat{u}(s)=\exp \left(\frac{z}{v} s+\frac{k_{1} z}{v}-\frac{m}{s+k_{2}}\right) \hat{y}(s)
$$

which, back in the time domain, yields an explicit formula using a Bessel function and a compact support convolution (see [16]). This formula provides a straightforward open-loop control law: given histories for the output 
temperature, one can simply determine the corresponding inlet temperature histories.

In practical applications, the true dynamics of the DOC systems is not simply a temperature-gas transport pass a solid monolith. In facts, the control variable is not the temperature, but, equivalently, the injected mass of fuel. These reductants are oxidized at the entry of the DOC system and, in turn, generate heat. One can model this heat generation using the mobile interface scheme of Figure 4. In fact, the DOC consists of two zones. An upstream reactive zone, and a temperature transport zone. The length of the (upstream) reactive zone directly depends on the amount of reductants under consideration, which is a control variable. It thus varies with the operating point. In turn, the complementary downstream transport zone also has a variable length.

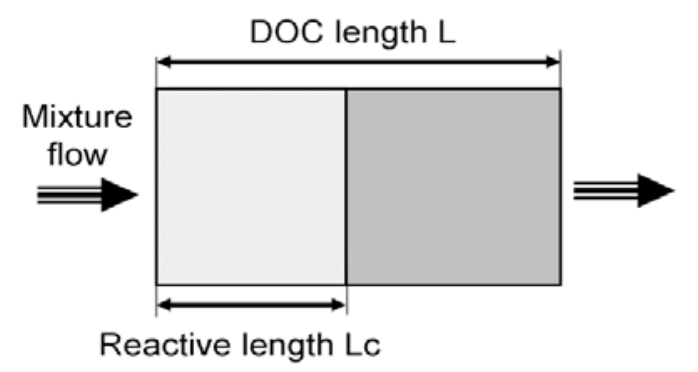

Fig. 4. DOC heat release model: reductants are spatially uniformly oxidized on the upstream part of the DOC. The system can be split into two zones separated by a moving interface: a reactive zone and a transport zone.

The location of the mobile interface can be identified quite accurately on experimental data. In practice, it is of great importance to account for the location of the interface in the derivation of control strategies. In particular, linear controllers reveal themselves to be efficient so long as they incorporate this variability in the computation of the gain scheduling and feed-forward actions [16, 17]. The reader can refer to [16, 18, 15] for practical vehicle applications relying on this model.

\section{A Nonlinear Stefan Problem}

In this second example, we study a heat diffusion equation with an endothermic reaction on a varying length. This can be seen as a crystal growth problem. Here, as in the previous example, the location of the mobile interface also depends on the control variable, but less directly, through the whole system dynamics. 
In the papers [7, 8] it has been shown how to calculate open-loop trajectories for a nonlinear Stefan problem. It is a system governed by a nonlinear parabolic partial differential equation which has been vastly studied from a numerical analysis point of view, i.e. to compute solutions for future times knowing initial conditions and future control actions. We desire to solve the inverse problem, i.e. knowing the behavior of the free boundary a priori we seek a solution, here as a convergent series, to calculate the control and description trajectories between two stationary states.

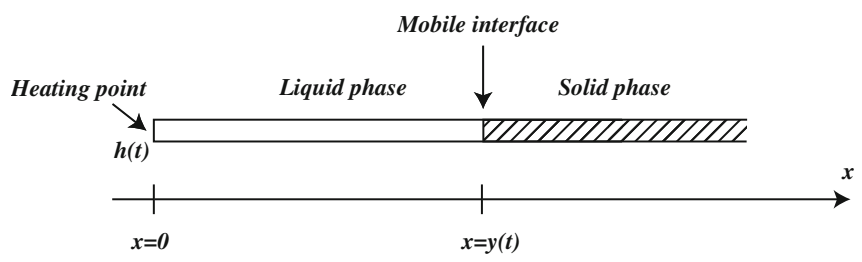

Fig. 5. Stefan problem with boundary control. Liquid phase with boundary control governed by a reaction-diffusion nonlinear partial differential equation in contact with a solid phase.

The classic Stefan problem considers a liquid phase column in contact at 0 degrees with an infinite phase solid, as shown in Figure 5. This problem is presented in detail in [1]. A list of problem reducing to this one can be found in 22] (including many processes formation and melting of crystals). Here, the Stefan problem is amended by adding a diffusion term and a nonlinear reaction term. This is a simplified model of reactant coolant fluid surrounded by solid phase.

Note $(x, t) \mapsto u(x, t)$ the temperature in the liquid phase, and $t \mapsto y(t)$ the varying location of the liquid/solid interface. The mappings $h(t)$ and $\psi(x)$ are the temperature on the fixed boundary $(x=0)$ and the initial condition, respectively $(t=0)$. The nonlinear Stefan problem consists in finding $u(x, t)$ and $y(t)$, for given $h(t)$ and $\psi(x)$ satisfying

$$
\left.\begin{array}{ll}
u_{t}=u_{x x}-\nu u_{x}-\rho u^{2}, & \forall(x, t) \in D_{T} \\
u(0, t)=h(t) \geq 0, & 0<t \leq T \\
u(x, 0)=\psi(x) \geq 0, & 0 \leq x \leq y(0) \\
u(y(t), t)=0, u_{x}(y(t), t)=-\dot{y}(t), & 0<t \leq T
\end{array}\right\}
$$

with

$$
D_{T} \equiv\{(x, t): 0<x<y(t), 0<t \leq T\}
$$

where the boundaries are noted

$B_{T} \equiv\{(0, t): 0<t \leq T\} \cup\{(x, 0): 0 \leq x \leq y(0)\} \cup\{(y(t), t): 0<t \leq T\}$ 


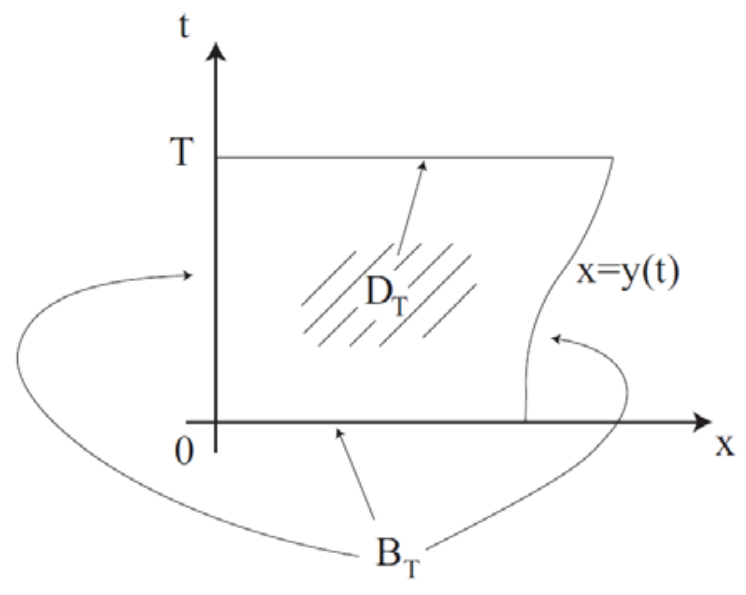

The boundary condition $u_{x}(y(t), t)=-\dot{y}(t)$ expresses that the heat flux at the interface is used for melting (or crystallization) of the solid phase. The parameters of conductivity and heat latent liquefaction are standard here, but without limitation, one may consider any factors in changes of variables $x$ and $t$.

The inverse problem is to calculate the boundary control $h(t)$ allowing the transition between two stationary states. As noted in [11, it is a non-Cauchy problem characteristic with the Cauchy data. This nonlinear problem can be solved by the following method. One can seek solutions (4) under the form of the following series

$$
u(x, t)=\sum_{n=0}^{\infty} \frac{a_{n}(t)}{n !}[x-y(t)]^{n}
$$

where the coefficients $\left(a_{n}(t)\right)$ satisfy the induction relations which are necessary and sufficient

$$
a_{n}=\dot{a}_{n-2}-a_{n-1} \dot{y}+\nu a_{n-1}+\rho \sum_{k=0}^{n-2}\left(\begin{array}{c}
n-2 \\
k
\end{array}\right) a_{n-2-k} a_{k}
$$

for $n \geq 2$, with $a_{0}=0($ from $u(y(t), t)=0)$ and $a_{1}=-\dot{y}\left(\right.$ from $-u_{x}(y(t), t)$ $=\dot{y}(t))$.

By increments, one can show that the series (5) is absolutely convergent where there exists strictly positives parameters $M, R, T$ such that

$$
\left|y^{(l+1)}(t)\right| \leq M \frac{l^{!^{\alpha}}}{R^{l}}, \forall l=0,1,2, \ldots, \forall t \in[0, T]
$$

A lower limit to its radius of convergence can be easily determined. The main difficulties lie in the calculation of recurrence bounds on the successive derivatives of the coefficients $\left(a_{n}(t)\right)$. This involves development of 
combinatorial derived cross terms from the nonlinear in $u^{2}$, for which one can use Chu-Vandermonde inequalities (see [21]). The lower bound on the radius of convergence is then calculated by analysis of roots of a polynomial of third degree. This lower bound can justify the use of this solution as series to solve the inverse problem of melting (or crystallization) of the solid phase by the control $h(t)$.

Suppose the liquid phase has an initial length $L$ and that we wish to reach the length $L+\Delta L$ in finite time. It is a challenging problem because the actuator $h(t)$ is located at the opposite end fixed the liquid-solid interface which will move over time. The control must compensate the energy loss due to melting solid and that due to diffusion and reaction term. To solve this problem, simply use the function

$$
y(\tau)= \begin{cases}L+\Delta L & \text { if } \tau \geq T \\ L+\Delta L g(\tau / T) & \text { if } T>\tau>0 \\ L & \text { if } \tau \leq 0\end{cases}
$$

where

$$
g(\tau)=\frac{f(\tau)}{f(\tau)+f(1-\tau)}, \tau \in[0,1],
$$

and

$$
f(\tau)= \begin{cases}e^{-\frac{1}{\tau}} & \text { if } \tau>0 \\ 0 & \text { if } \tau \leq 0\end{cases}
$$

This function defines a smooth transition between the lengths $L$ and $L+\Delta L$. By choosing the parameter $T$ depending on other physical parameters, one can guarantee that the radius of convergence of the series is larger than $L+\Delta L$ proving that that the series expansion, and therefore the solution to the inverse problem are valid.

This work follows [19] on reaction diffusion equation with fixed boundary. Besides convergence of this series for a very special class of Gevrey functions (as defined in [1]) used in an explicit assumption depending on physical parameters of the system, one can also prove a maximum principle stating that the maximum temperature is always achieved on the sides of the domain 8 . Asymptotic positivity property of the solution can also be established.

\section{Mixing Models}

We now pursue our panorama of distributed systems with mobile interfaces by considering mixing systems 1 . In this case, a rather unusual model can be

\footnotetext{
${ }^{1}$ The interested reader can refer to 20, 2] treating the related problem of blending
} systems. 
proposed, where the motion of the moving interface separating a homogeneous zone and a distributed zone depends on the derivative of the input signal.

We expose ways to model mixing phenomena for Newtonian fluids under unsteady stirring conditions in agitated vessels using helical ribbon impellers. A model of torus reactor including a well-mixed zone and a transport zone is considered. The originality of the arrangement of ideal reactors developed in 5, 6] lies in the time-dependent location of the boundaries between the two zones. Interestingly, this concept is applied to model the positive influence of unsteady stirring conditions on homogenization process. It appears that this model allows the easy derivation of a control law, which is a great advantage when optimizing the dynamics of a mixing process. We now detail this model.

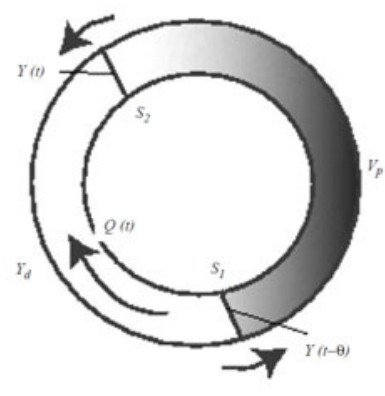

Fig. 1. Sketch of torus model proposed in this study.

Fig. 6. Sketch of torus model proposed in [5, 6]

The mixing system is as follows. Consider a torus of fixed volume $\mathrm{V}$ divided into two ideal reactors (a constant stirred tank reactor of volume $V_{d}$ and a plug flow zone of volume $V_{p}=V-V_{d}$ ) in which flows a Newtonian fluid with a uniform time-varying flow rate $\dot{Q}$ in a clockwise direction (see Figure 6). Further, $y$ refers to the fluid concentration $(\mathrm{kg} / \mathrm{m} 3)$ which varies with time and space. It is assumed that the total material quantity of the component $y$ in the reactor remains constant. The originality of the torus reactor arises from the time-dependent position of the interfaces ( $\mathrm{S} 1$ and $\mathrm{S} 2$ ) which separate the two ideal flow zones. Indeed, it is assumed that S1 and S2 move alternately in a counter-clockwise direction to the flow rate fluctuations. Consequently, when the flow rate is non-steady, the volumes $\left(V_{d}\right.$ and $\left.V_{p}\right)$ of the two ideal reactors are time variant. In particular, it is assumed that S1 (respectively, S2) moves only when positive (respectively, negative) variations in the flow rate occur in the torus volume and is otherwise motionless. Note also, that when a variation of flow rate occurs, not only the volumes of the zones vary but their location within the torus evolves counter-clockwise. We assume that at each time $t$ the flow rate $\dot{Q}(t)$ is proportional to the impeller rotational speed $N(t)$. For steady operations simulation results obtained with this model are close to 


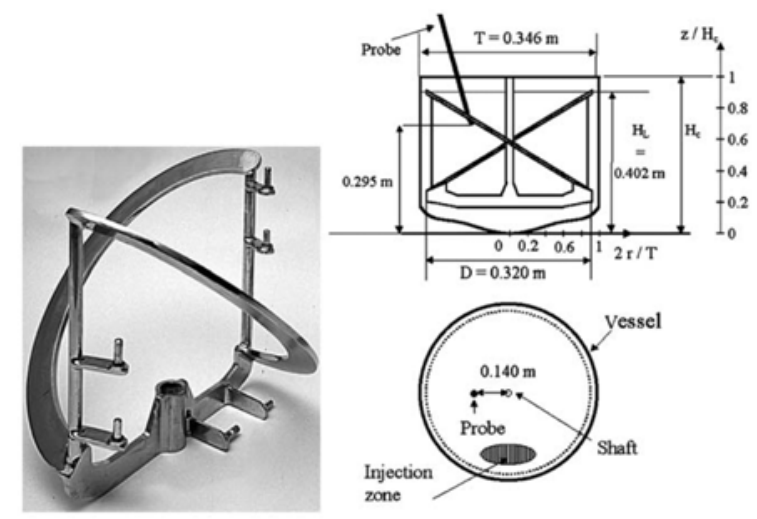

Fig. 7. A mixing vessel used for experimental validation of the proposed mixing model, from [6]

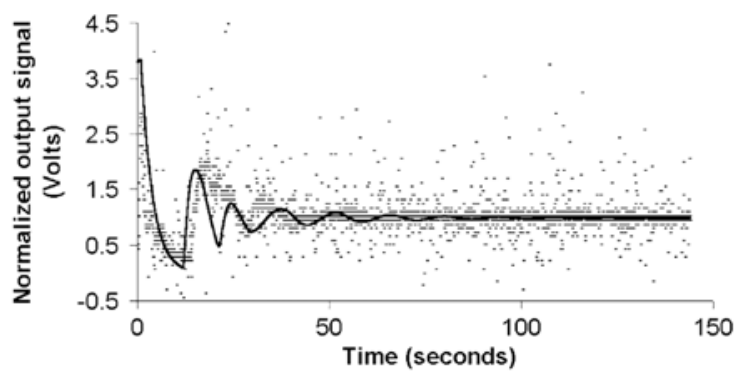

Fig. 8. The torus model reproduces well the experimentally observed mixing measured by a conductivity probe, from 6

the reference results [14. In the case of unsteady stirring, the model accounts for the experimental observation that an improvement in mixing occurs when a positive variation in the rotational speed is enforced. For example, in the case of a positive variation in impeller rotational speed, the volume of the stirred tank reactor increases while that of the plug flow decreases. As the whole volume of the torus loop is unchanged, an enhancement in mixing is expected.

Note $\dot{V}_{d}^{+}$(respectively, $\dot{V}_{d}^{-}$) the variation of volume $V_{d}$ due to the motion of $\mathrm{S} 1$ (resp., S2) in the torus, and let $\theta$ be the residence time of the particle leaving the plug flow zone at time $t$. Then, the whole system can be characterized by the following differential equations 


$$
\begin{aligned}
& V=V_{d}(t)+V_{p}(t) \\
& \int_{t-\theta}^{t} \dot{Q}(\sigma) d \sigma=V-V_{d}(\dot{Q}(t-\theta))-\int_{t-\theta}^{t} \dot{V}_{d}^{+}(\sigma) d \sigma \\
& V_{d}(\dot{Q}(t)) \frac{d(y(t))}{d t}=\left(\dot{Q}(t)+\dot{V}_{d}^{+}\right)(y(t-\theta)-y(t)), \\
& \dot{Q}(t)=\alpha N(t)
\end{aligned}
$$

with

$$
\begin{aligned}
& \dot{V}_{d}^{+}=k \frac{d N}{d t}, \text { if } \frac{d N}{d t}>0, \dot{V}_{d}^{+}=0 \text { otherwise } \\
& \dot{V}_{d}^{-}=-k \frac{d N}{d t}, \text { if } \frac{d N}{d t}<0, \dot{V}_{d}^{+}=0 \text { otherwise. }
\end{aligned}
$$

This model represents experimental data well. To check its validity, a mixing vessel pictured in Figure 7 was used. The agitated fluid is an aqueous solution of glucose. The rotational speed was controlled to reproduce increasing and decreasing ramps. A conductivity probe was used to obtain the circulation curves in the vessel. The rotational speed and the conductivity signal were recorded throughout the mixing process. The values of the rotational speed varied from 0.16 to $1.5 \mathrm{rev} / \mathrm{s}$. Mixing and circulation times were determined from the response signal recorded after tracer injection. As is pictured in Figure 8, for the experimental conditions tested, the probe conductivity measurements are in close agreement with the expected behavior reproduced by the model.

\section{Multiphasis Slug Flow}

Finally, we wish to complete our catalogue of distributed systems with moving interfaces with a problem of multiphase flow. This problem is of great importance in the oil industry where long pipes (named risers, or flowlines) are used to transport large blends of gas, oil and water. The gas and the liquid phase do not mix, and, in the case when the dispersed bubbles gather, they form large bubbles, named "slugs" which induce malicious pressure variations which are highly detrimental for industrial facilities. In such cases, the interface is the boundary between liquid and gas phase. It is indirectly controlled by remote inputs.

In details, risers are long pipes connecting reservoirs to surface facilities for oil production. Severe slugging is a flow regime that arises mostly when entering tail production of an oil field. It is characterized by an unstable multiphase flow, where slugs of liquid accumulate before being pushed upwards by the gas. It is also characterized by oscillations of the pressure in the pipeline and oscillations of flow rates of gas and oil at the production end of the pipe. Although the phenomenon itself can be observed and sometimes 
reproduced on test rigs, its causes are not always known. Because the severe slugging flow regime can damage the installations (and most importantly reduce the oil production), various techniques have been investigated in view of suppressing it. The riser length typically ranges from a few hundred meters to several kilometers. To avoid instability, the most straightforward technique

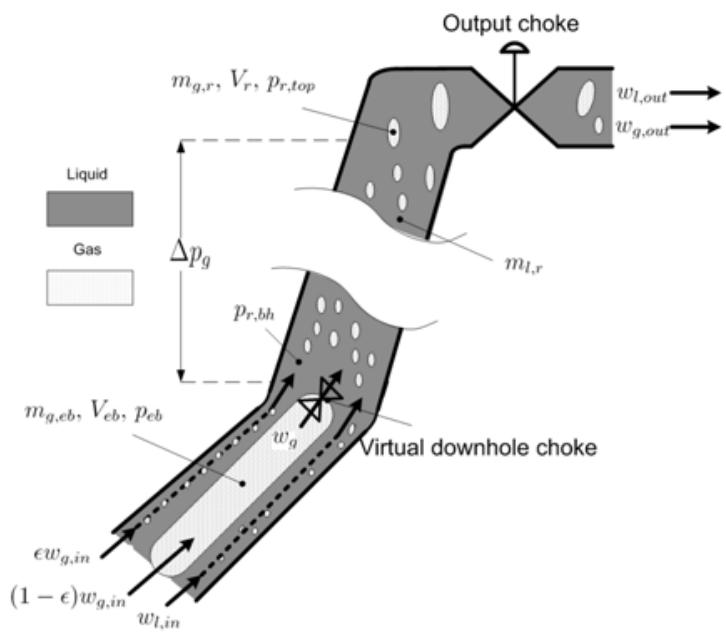

Fig. 9. A vertical riser carrying a multiphase flow, from 3]. An elongated bubble located at the bottom of the riser is subjected to a pressure buildup until it is released and generate a slugging flow. The interface between this elongated bubble and the rest of the riser is a virtually controlled interface.

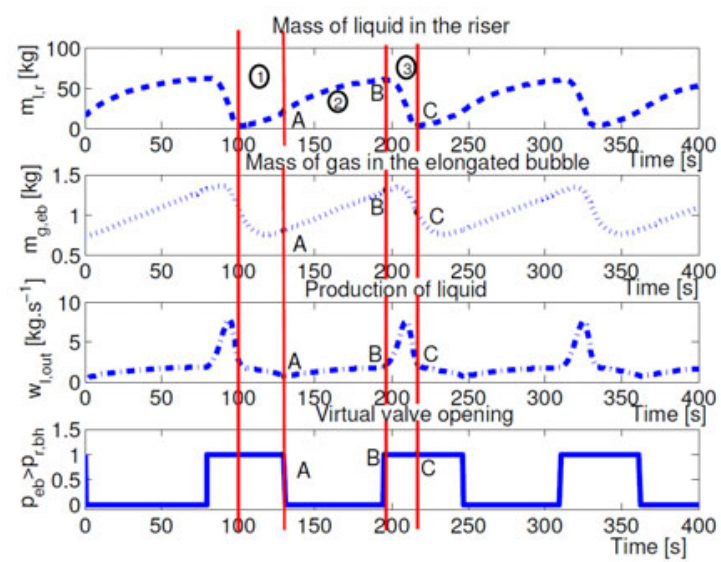

Fig. 4. The three stages of the oscillations

Fig. 10. The successive steps of the slugging cycle reproduced by the simple model 
consists in choking down manually the pipes thanks to a choke located upstream the separator. Although this solution stabilizes the flow, it reduces significantly the oil production which, in turn, motivates the investigation of dynamic control of the valve. Indeed, it is possible, in closed-loop, to stabilize at higher flow rates.

Two classes of models can be found in the literature. The most accurate type is based on (usually nonlinear) partial differential equations representing two-phase (oil and gas) or three-phase (oil, water and gas) flows. These models are able to reproduce the slugging phenomenon in many cases, but fail to match the behavior of real-life wells in other cases, in particular when the instability comes from reservoir dynamics, for which there is little knowledge about. Unfortunately, it is not possible to derive control laws from these models because of their complexity. The second class of models is based on ordinary differential equations and represents a different trade-off between accuracy and complexity. A prime example is the model presented in [13, which, besides its numerous merits, does not sufficiently rely on physics to accurately reproduce the physical response of the system. Finally, the model is not general enough and is designed for a specific geometry. This is also the case for the model of gas-lift presented in [23, 26, 25].

Consider a vertical riser subjected to a constant input flow. The output flow of the riser is controlled by a choke. Unstable flow regime can occur, especially when the choke is largely opened, which, unfortunately, corresponds to a point of industrial interest. This kind of instability is also observed on related systems: oil wells with a gas reservoir [28, 12, risers with low-point [27, 10, 9]. Generally, switches of valves are reported to be at the birth of the oscillating phenomena: downhole choke plays a key role in the casing-heading in [23, [12, 24], while the geometric low-point acting as a valve is studied in [27]. In the riser considered here, no such valve exists or is even suggested by the geometry. Yet, one can model the riser using a virtual choke located at a well chosen point at the bottom of the riser. In this approach, the riser is modeled as a three-state set of ordinary differential equations. As is detailed in [3], one can tune the model analytically to fit most physical systems of interest. The equations reproduce the slugging flow regime as follows. The elongated bubble is subjected to a pressure buildup until its pressure get high enough so that the bubble is eventually released and travels through the rest of the vertical riser and produced a slugging flow regime. Then, the pressure buildup starts over again. The successive steps of this cycle are pictured in Figure 10, Further, this model suggest a control design that allows to stabilize the flow. One can refer to [3,4] for an experimental study and a mathematical derivation of this control law.

\section{Conclusion}

In this paper, several distributed parameter systems with an internal mobile interface have been presented. In each case, the governing equations are 
relatively simple, and it appears that the introduction of a mobile interface is a key feature to make this model realistic. At various levels, the location or the nature of the mobile interface can be controlled by the input variables. In the case of the DOC system, it is directly dependent on the amount of reductants entering the system. In the Stefan problem, the liquid-solid interface moves as the heat flux travels through the whole system. In the mixing vessel, the interface moves according to sign of the time variations of the rotation speed of the blender. In the multiphase flow, the interface is virtually actuated by a the histories of the control variable. Interestlingly, all these models are simple enough to provide direct insight into the solution of control problems of engineering interest: thermal response of the DOC system, inverse control of crystal growth, optimization of blending dynamics, stabilization of slugging flows. The interested reader can refer to [16, 8, 6, 3] and the references therein for details.

Acknowledgements. The author wishes to thank several colleagues and associates who have greatly contributed to the works presented in this article: in alphabetical order, Y. Creff, O. Lepreux, F. di Meglio, L. Sinègre.

\section{References}

1. Cannon, J.R.: The one-dimensional heat equation. Encyclopedia of Mathematics and its applications, vol. 23. Addison-Wesley Publishing Company, Reading (1984)

2. Chèbre, M., Creff, Y., Petit, N.: Feedback control and optimization for the production of commercial fuels by blending. Journal of Process Control 20(4), 441-451 (2010)

3. Di Meglio, F., Kaasa, G.-O., Petit, N.: A first principle model for multiphase slugging flow in vertical risers. In: Proc. of the 48th IEEE Conf. on Decision and Control (2009)

4. Di Meglio, F., Kaasa, G.-O., Petit, N., Alstad, V.: Model-based control of slugging flow: an experimental case study. In: Proc. of the, American Control Conference (to appear 2010)

5. Dieulot, J.-Y., Petit, N., Rouchon, P., Delaplace, G.: An arrangement of ideal zones with shifting boundaries as a way to model mixing processes in unsteady stirring conditions in agitated vessels. Chemical Engineering Science 60(20), 5544-5554 (2005)

6. Dieulot, J.-Y., Petit, N., Rouchon, P., Delaplace, G.: A torus model containing a sliding well-mixed zone as a way to represent mixing process at unsteady stirring conditions in agitated vessels. Chemical Engineering Communications 192, 805-826 (2005)

7. Dunbar, W.B., Petit, N., Rouchon, P., Martin, P.: Boundary control for a nonlinear Stefan problem. In: Proc. of the 42nd IEEE Conf. on Decision and Control (2003)

8. Dunbar, W.B., Petit, N., Rouchon, P., Martin, P.: Motion planning for a nonlinear Stefan problem. ESAIM: Control, Optimisation and Calculus of Variations 9, 275-296 (2003) 
9. Duret, E.: Dynamique et contrôle des écoulements polyphasiques. PhD thesis, École des Mines de Paris (2005)

10. Henriot, V., Duret, E., Heintz, E., Courbot, A.: Multiphase production control: Application to slug flow. Oil \& Gas Science and Technology 57(1), 87-98 (2002)

11. Hill, C.D.: Parabolic equations in one space variable and the non-characteristic Cauchy problem. Comm. Pure Appl. Math. 20, 619-633 (1967)

12. $\mathrm{Hu}, \mathrm{B}$.: Characterizing gas-lift instablilities. $\mathrm{PhD}$ thesis, Norwegian University of Science and Technology (2004)

13. Kaasa, G.-O.: Attenuation of slugging in unstable oil by nonlinear control. In: Proc. of the 17th IFAC World Congress (2008)

14. Khang, S.J., Levenspiel, O.: New scale-up and design method for stirrer agitated batch mixing vessels. Chemical Engineering Science 31, 569-577 (1976)

15. Lepreux, O.: Model-based Temperature Control of a Diesel Oxidation Catalyst. PhD thesis, École des Mines de Paris (2009)

16. Lepreux, O., Creff, Y., Petit, N.: Motion planning for a Diesel oxidation catalyst outlet temperature. In: Proc. of the 2008 American Control Conference (2008)

17. Lepreux, O., Creff, Y., Petit, N.: Model-based control design of a diesel oxidation catalyst. In: ADCHEM 2009, International Symposium on Advanced Control of Chemical Processes (2009)

18. Lepreux, O., Creff, Y., Petit, N.: Warm-up strategy for a diesel oxidation catalyst. In: Proc. of European Control Conf. 2009 (2009)

19. Lynch, A.F., Rudolph, J.: Flatness-based boundary control of a nonlinear parabolic equation modelling a tubular reactor. In: Isidori, A., LamnabhiLagarrigue, F., Respondek, W. (eds.) Lecture Notes in Control and Information Sciences 259: Nonlinear Control in the Year 2000, vol. 2, pp. 45-54. Springer, Heidelberg (2000)

20. Petit, N., Creff, Y., Rouchon, P.: Motion planning for two classes of nonlinear systems with delays depending on the control. In: Proc. of the 37th IEEE Conf. on Decision and Control, pp. 1007-1011 (1998)

21. Petkovsek, M., Wilf, H.S., Zeilberger, D.: A=B, Wellesley (1996)

22. Rubinstein, L.I.: The Stefan problem. Translations of mathematical monographs, vol. 27. AMS, Providence (1971)

23. Sinègre, L.: Dynamic study of unstable phenomena stepping in gaslift activated systems. PhD thesis, École des Mines de Paris (2006)

24. Sinègre, L., Petit, N., Lemétayer, P., Gervaud, P., Ménégatti, P.: Casingheading phenomenon in gas-lifted well as a limit cycle of a $2 \mathrm{~d}$ model with switches. In: Proc. of the 16th IFAC World Congress (2005)

25. Sinègre, L., Petit, N., Ménégatti, P.: Predicting instabilities in gas-lifted wells simulation. In: Proc. of the 2006 American Control Conference (2006)

26. Sinègre, L., Petit, N., Saint-Pierre, T.: Active control strategy for density-wave in gas-lifted wells. In: Proc. of the ADCHEM 2006, International Symposium on Advanced Control of Chemical Processes (2006)

27. Storkaas, E.: Control solutions to avoid slug flow in pipeline-riser systems. PhD thesis, Norwegian University of Science and Technology (2005)

28. Torre, A.J., Blais, R.N., Brill, J., Doty, D., Schmidt, Z.: Casing-heading in flowing wells. In: SPE Production Operations Symposium, no. SPE 13801 (1987) 